\title{
The effect of experimental muscle pain on the background electrical brain activity
}

\author{
Phanomporn Veerasarn and Christian S. Stohler \\ Stomatognathic Physiology Laboratory, School of Dentistry, University of Michigan, Ann Arbor, MI 48109-1078 (USA) \\ (Received 19 June 1991, revision received 22 November 1991, accepted 29 November 1991)
}

\begin{abstract}
Summary The purpose of this project was to investigate whether specific effects in the background activity of the brain associated with the experience of pain can be depicted by means of quantitative electroencephalography (EEG). Lasting pain was induced by intramuscular infusion of hypertonic saline. The infusion was titrated to maintain pain for a sufficient time to obtain enough data for meaningful analysis. In a first study on 12 subjects, using a single, blind, repeated measures design with randomization of the administration of isotonic $(0.9 \%)$ and hypertonic $(5 \%)$ saline, and with subjects unaware of the fact that one substance was isotonic saline, a statistically significant pain response could be attributed to the administration of hypertonic saline. In a second study on 19 subjects, again using a randomized repeated measures design, topographic EEG measures were examined with respect to experimentally induced pain and pain from memory. Prior to each of these experimental stages, baseline recordings were obtained to satisfy the requirement of the crossover design. In addition to the common frequency bands used in EEG, we also obtained data in the frequency range of $35-100 \mathrm{~Hz}$. The short-term variability of the selected EEG measures and their suitability as a sample estimate were assessed by computing the coefficient of variation from all selected epochs of a given subject at baseline. When compared to baseline, spectral analyzed EEG measures during experimental pain demonstrated statistically significant increases in the beta and $35-100 \mathrm{~Hz}$ frequency ranges, most notably at the temporal recording sites. There was no statistically significant difference between the EEG measures for (1) experimental pain vs. pain from memory, and (2) the 2 baseline recordings. The great variability in the topographical aspect of the between-subject response was interpreted as being strongly suggestive of the contamination of EEG measures by phenomena attributed to the jaw, facial and scalp musculature. In fact, Pearson correlation coefficients, as high as 0.92 , were found between measures in the frequency band of $35-100 \mathrm{~Hz}$ and the beta frequency range. The unexplained variance in the heightened beta cortical power density can be attributed to the vigilance scanning of pain processes. Due to the fact that the statistically significant effect of pain on the topographic EEG measures were not different from imagined pain, we concluded that these effects are non-specific for pain.
\end{abstract}

Key words: Pain; Electroencephalography

\section{Introduction}

Many attempts have been made to apply neurophysiologic and psychophysiologic methods to quantify the subjective experience of human pain. Specifically, research in assessing human pain responses through

Correspondence to: Dr. Christian S. Stohler, Stomatognathic Physiology Laboratory, School of Dentistry, University of Michigan, Ann Arbor, MI 48109-1078, USA. analyses of (1) brain evoked potentials and (2) background brain electrical activity has gained considerable interest in the past decades.

A number of reports suggest that brain evoked potentials correlate with stimulus intensity and pain perception (Chatrian et al. 1975; Carmon et al. 1976, 1978; Harkins and Chapman 1978; Chapman et al. 1979, 1981; Chen et al. 1979; Bromm 1985). However, not only noxious stimulation but also the state of arousal, attention, and increased activation of nonnociceptive afferents are related to an increase in 
evoked potential amplitude (Velasco et al. 1973; Desmedt and Debecker 1979; Chudler and Dong 1983; Cruccu et al. 1983; Leandri ct al. 1985). Therefore, the utility of brain evoked potentials as an index of pain experience has not been accepted.

The second line of research is concerned with the resting brain activity by means of quantitative electroencephalography (EEG). It has been reported that brain electrical activity correlates with clinical and experimental pain (Chen et al. 1981, 1983, 1984, 1987 , 1989; Dworkin et al. 1984; Manna et al. 1984; Drake et al. 1988). Specifically, analyses of EEG power spectra have shown that EEG wave form, amplitude and frequency components can be influenced by pain. In a recent study by Chen et al. (1989), using the cold-pressor test, an increase in cortical beta power density as a direct correlate of the subjective pain has been reported. However, whether this response is specific for pain needs to be determined.

Consequently, the aim of our project was to examine (a) whether we are able to replicate earlier findings of a statistically significant effect of pain on the topographic EEG measures, and (b) if true, to examine whether these effects are specific for pain.

\section{Methods}

This project consisted of 2 studies. The lst study (Study 1) examined hypertonic $(5 \%)$ saline as an agent to induce lasting pain. The 2nd study (Study 2) determined the effects in the background brain electrical activity in association with experimental pain and imagined pain. Imagined pain was used to control for non-specific effects in the psychological state.

\section{Subjects}

All subjects gave consent and were informed about the experimental procedures and the fact that they could withdraw from the experiment involving experimental pain at any time. Subjects with neurological illness and major medical history were excluded by a screening questionnaire. Accepted subjects were pain free on the day of the experiment; none was on any medication. Experiments were approved by the Institutional Committee for Human Subject Use.

\section{Study 1}

Twelve (12) healthy young adults ( 5 females, 7 males), aged 22-37 years (mean age: 25 years; S.D. $=4.3$ years) served as paid subjects $(\$ 50)$ in an experiment aimed to characterize the pain stimulus to be used in Study 2.

\section{Study 2}

Nineteen (19) healthy young adults ( 2 females, 17 males), aged 21-38 years (mean age: 26.5 years; S.D. $=4.7$ years), participated in an experiment, addressing the questions (a) whether a statistically significant response in the topographic EEG measures can be detected in pain, and (b) if any, whether the effect is specific for the condition of noxious input. Each volunteer was paid $\$ 75$ for participation.

\section{Experimental design}

\section{Study I}

Using a single. blind, repeated measures design with randomization of the sequence of substance administration, we investigated whether hypertonic saline was different from isotonic saline in as far as the pain intensity response was concerned. Subjects were informed that 2 pain-causing agents were being compared.

\section{Study 2}

For each subject, experiments were performed in a single session using a controlled crossover and repeated measures design. The experiment consisted of 4 stages: (1) baseline 1, (2) noxious stimulation (experimental jaw muscle pain), (3) baseline 2. and (4) sham pain. Subjects were randomly assigned the sequential order of the experimental stages, i.e., noxious stimulation preceded sham pain or vice versa.

\section{Experimental protocol}

\section{Study 1}

Hypertonic or isotonic saline $(0.1 \mathrm{ml})$ was injected into the belly of the masseter muscle in the center between origin and insertion, and the anterior and posterior borders of the muscle. The sequence of administration of either the hypertonic or isotonic saline was randomized. The first injection always occurred on the right side and the second on the left side. Subjects were asked to rate the perceived pain intensity using a computerized visual analog scale (VAS).

\section{Study 2}

The experimental stages were defined as follows.

Baseline I. Subjects were asked to sit quietly in a reclined dental chair with both eyes closed and to relax but also be alert.

Noxious stimulation. Experimental jaw muscle pain was induced by injections of hypertonic (5\%) saline into the body of the masseter muscle (Stohler et al. 1991; Zhang et al. submitted). Leaving the needle in place, repeated injections of hypertonic saline were used to obtain sufficient data for meaningful analysis. With both eyes closed, subjects were instructed to rate the perceived pain intensity on a 1(1)-point scale by using their fingers and update their pain intensity whenever it changed.

Sham pain. Imagined pain was used to control for non-specific reactions to pain. Subjects were asked to recall the earlier experimental pain or a past painful experience in case sham pain preceded experimental pain. Rating techniques for pain intensity from memory were identical to those of the noxious stimulation stage.

Baseline 2. This was the stage following a period of at least $3 \mathrm{~min}$ of zero pain intensity after either experimental or sham pain. The instructions to the subject were similar to baseline 1.

\section{Procedures}

\section{Study 1}

A 100 -mm-long electronic display, containing 50 light-emitting diodes (LEDs), was used as VAS. A computer-generated ramp function was cmployed to turn on the LEDs sequentially in increasing order until all were lit. The ramp, which lasted for $10 \mathrm{sec}$, was initiated at $15-\mathrm{sec}$ intervals. The subject was given a thumb switch to indicate his/her present pain intensity at the time when the LED corresponding to the present perceived pain intensity level lighted in each ramp cycle. VAS scores and the elapsed time were stored in a data file (Stohler et al. 1991; Zhang et al. submitted). 
Study 2

Experiments were performed in a radiofrequency-shielded room. Scalp recorded bioelectrical activity was obtained using the Cadwell S32 encephalograph (Cadwell Laboratories, Inc., Kennewick, WA 99336). Surface electrodes were carefully attached to the frontal (F3, F4), temporal (T3, T4), parietal (P3, P4), and occipital (O1, O2) recording sites according to the International 10-20 System with reference to the linked ear lobes. An electrode impedance of less than $5 \mathrm{~kW}$ in each lead was acceptable. To monitor eye movement, an additional electrode was attached to the lower left eyelid. Bandpass filtering between 0.5 and $100 \mathrm{~Hz}$ was performed. Data were digitized at a rate of $300 \mathrm{~Hz} /$ channel in agreement with the Nyquist theorem for signal sampling. For each experimental stage, topographic EEG measures were obtained for at least $7 \mathrm{~min}$. Data were

TABLE I

RESULTS OF $F$ TESTS OF REPEATED MEASURES ANOVA, CALCULATED FOR EXPERIMENTAL SEQUENCE, HEMISPHERES, AND EXPERIMENTAL STAGES FOR EACH TOPOGRAPHIC LOCATION

\begin{tabular}{|c|c|c|c|c|c|c|c|c|c|c|c|c|}
\hline \multirow[t]{3}{*}{ Sources } & \multicolumn{12}{|c|}{ EEG measures } \\
\hline & \multicolumn{2}{|l|}{ Delta } & \multicolumn{2}{|c|}{ Theta-Alpha } & \multicolumn{2}{|l|}{ Beta } & \multicolumn{2}{|c|}{$35-100 \mathrm{~Hz}$} & \multicolumn{2}{|c|}{$0.5-100 \mathrm{~Hz}$} & \multicolumn{2}{|c|}{ Mean frequency } \\
\hline & $(F)$ & $(P)$ & $(F)$ & $(P)$ & $(F)$ & $(P)$ & $(F)$ & $(P)$ & $(F)$ & $(P)$ & $(F)$ & $(P)$ \\
\hline \multicolumn{13}{|l|}{ Frontal $(F 3, F 4)$} \\
\hline \multicolumn{13}{|l|}{ Between subjects } \\
\hline Sequence & 0.780 & 0.389 & 0.330 & 0.573 & 11.228 & $0.004^{*}$ & 4.713 & $0.044^{*}$ & 4.240 & 0.055 & 1.953 & 0.180 \\
\hline \multicolumn{13}{|l|}{ Within subjects } \\
\hline Hemisphere & 35.281 & $0.000 *$ & 0.786 & 0.388 & 3.189 & 0.092 & 1.179 & 0.293 & 0.185 & 0.672 & 3.776 & 0.069 \\
\hline Hemisphere $\times$ Sequence & 0.040 & 0.844 & 0.066 & 0.801 & 0.076 & 0.786 & 0.238 & 0.632 & 0.072 & 0.792 & 0.530 & 0.476 \\
\hline Stage & 0.604 & 0.579 & 2.103 & 0.138 & 4.740 & $0.021^{*}$ & 6.934 & $0.011^{*}$ & 5.703 & $0.018 *$ & 16.223 & $0.000^{*}$ \\
\hline Stage $\times$ Sequence & 0.242 & 0.822 & 0.924 & 0.406 & 2.188 & 0.137 & 2.093 & 0.159 & 2.449 & 0.123 & 1.766 & 0.190 \\
\hline Hemisphere $\times$ Stage & 4.196 & $0.012 *$ & 0.954 & 0.357 & 0.359 & 0.637 & 1.975 & 0.162 & 1.537 & 0.233 & 2.690 & 0.083 \\
\hline Hemisphere $\times$ Stage $\times$ Scquence & 1.576 & 0.211 & 0.979 & 0.351 & 0.138 & 0.807 & 1.470 & 0.246 & 0.688 & 0.481 & 1.451 & 0.249 \\
\hline \multicolumn{13}{|l|}{ Temporal $(T 3, T 4)$} \\
\hline \multicolumn{13}{|l|}{ Between subjects } \\
\hline Sequence & 1.921 & 0.184 & 0.075 & 0.787 & 1.055 & 0.319 & 0.745 & 0.400 & 1.017 & 0.327 & 0.080 & 0.780 \\
\hline \multicolumn{13}{|l|}{ Within subjects } \\
\hline Hemisphere & 0.431 & 0.520 & 2.411 & 0.139 & 5.388 & $0.033^{*}$ & 6.291 & $0.023^{*}$ & 6.220 & $0.023^{*}$ & 3.379 & 0.084 \\
\hline Hemisphere $\times$ Sequence & 0.024 & 0.879 & 0.596 & 0.451 & 0.406 & 0.532 & 0.571 & 0.460 & 0.575 & 0.459 & 1.125 & 0.304 \\
\hline Stage & 0.381 & 0.740 & 1.066 & 0.359 & 5.599 & $0.012^{*}$ & 7.704 & 0.005 & 6.675 & $0.008 *$ & 20.320 & $0.000^{*}$ \\
\hline Stage $\times$ Sequenee & 0.062 & 0.969 & 1.667 & 0.201 & 1.565 & 0.227 & 1.690 & 0.208 & 1.736 & 0.201 & 1.271 & 0.295 \\
\hline Hemisphere $\times$ Stage & 0.272 & 0.834 & 0.552 & 0.613 & 2.823 & 0.069 & 2.165 & 0.119 & 2.404 & 0.094 & 1.385 & 0.260 \\
\hline Hemisphere $\times$ Stage $\times$ Sequence & 0.343 & 0.782 & 0.192 & 0.864 & 0.961 & 0.397 & 2.327 & 0.101 & 1.883 & 0.158 & 4.823 & $0.006^{*}$ \\
\hline \multicolumn{13}{|l|}{ Parietal $(P 3, P 4)$} \\
\hline \multicolumn{13}{|l|}{ Between subjects } \\
\hline Sequence & 0.278 & 0.605 & 0.104 & 0.752 & 5.615 & $0.030^{*}$ & 0.100 & 0.756 & 0.397 & 0.537 & 0.004 & 0.950 \\
\hline \multicolumn{13}{|l|}{ Within subjects } \\
\hline Hemispere & 0.547 & 0.470 & 0.260 & 0.617 & 0.084 & 0.775 & 3.163 & 0.093 & 1.220 & 0.285 & 4.150 & 0.058 \\
\hline Hemisphere $\times$ Scquence & 0.054 & 0.819 & 2.283 & 0.149 & 9.554 & $0.007 *$ & 5.801 & $0.028^{*}$ & 7.112 & $0.016^{*}$ & $1.90 \$$ & 0.185 \\
\hline Stage & 0.280 & 0.779 & 2.497 & 0.086 & 3.799 & $0.044 *$ & 6.696 & $0.014^{*}$ & 4.876 & $0.028^{*}$ & 23.147 & $0.000^{*}$ \\
\hline Stage $\times$ Sequence & 0.556 & 0.596 & 0.862 & 0.446 & 1.617 & 0.219 & 1.590 & 0.225 & 1.778 & 0.196 & 2.147 & 0.147 \\
\hline Hemisphere $\times$ Stage & 1.574 & 0.222 & 0.438 & 0.711 & 0.917 & 0.411 & 0.361 & 0.708 & 0.706 & 0.508 & 0.689 & 0.520 \\
\hline Hemisphere $\times$ Stages $\times$ Sequence & 0.811 & 0.454 & 0.477 & 0.685 & 0.620 & 0.546 & 0.474 & 0.634 & 0.477 & 0.635 & 0.273 & 0.780 \\
\hline \multicolumn{13}{|l|}{ Occipital $(01, O 2)$} \\
\hline \multicolumn{13}{|l|}{ Between subjects } \\
\hline Sequence & 1.994 & 0.176 & 0.000 & 0.994 & 2.113 & 0.164 & 0.228 & 0.639 & 0.059 & 0.812 & 0.441 & 0.515 \\
\hline \multicolumn{13}{|l|}{ Within subjects } \\
\hline Hemisphere & 0.731 & 0.404 & 0.853 & 0.369 & 0.773 & 0.391 & 0.488 & 0.494 & 1.065 & 0.317 & 0.323 & 0.577 \\
\hline Hemisphere $\times$ Sequence & 1.506 & 0.236 & 0.594 & 0.451 & 0.330 & 0.573 & 1.731 & 0.206 & 0.028 & 0.868 & 3.949 & 0.063 \\
\hline Stage & 1.439 & 0.247 & 2.785 & 0.075 & 10.102 & $0.000 *$ & 12.142 & $0.000^{*}$ & 11.994 & $0.000^{*}$ & 24.798 & $0.000^{*}$ \\
\hline Stage $\times$ Sequence & 0.092 & 0.944 & 0.878 & 0.426 & 0.915 & 0.406 & 0.895 & 0.401 & 0.996 & 0.375 & 0.841 & 0.443 \\
\hline Hemisphere $\times$ Stage & 0.802 & 0.466 & 0.056 & 0.976 & 1.807 & 0.179 & 1.378 & 0.266 & 1.068 & 0.363 & 0.031 & 0.983 \\
\hline Hemisphere $\times$ Stages $\times$ Sequence & 0.477 & 0.641 & 0.593 & 0.607 & 0.994 & 0.382 & 1.481 & 0.241 & 1.200 & 0.317 & 1.543 & 0.223 \\
\hline
\end{tabular}

* Significant at $P<0.05$. 
stored on optical disk for further off-line analysis. After termination of the EEG recordings, subjects were asked to report the quality of pain and its spatial distribution.

\section{Data sampling and data analysis}

In Study 2, artifact screening and signal analysis were done off-line. The recorded EEGs were visually inspected, and epochs containing no visible artifacts were manually selected. Artifacts from physiologic sources such as muscle activity from swallowing, eye movement and those from non-physiologic sources (e.g., movement of electrodes and leads) were excluded. For each experimental stage, at least twenty-four mostly, however, thirty 2.5 -sec artifact-free epochs were chosen for analysis. In experimental pain, such sampling was performed for the condition of pain with an intensity of greater than 5 on the 10-point intensity scale.

Each selected epoch was subjected to Fast Fourier Transformation (FFT). The following parameters were computed: mean frequency $(\mathrm{Hz})$, total power $(\mathrm{Nw})$ in various frequency bands (delta: $0.5-3.5 \mathrm{~Hz}$, theta-alpha: $3.5-13 \mathrm{~Hz}$, beta: $13-35 \mathrm{~Hz}$, and the frequency range of $35-100 \mathrm{~Hz}$ ) of specified cortical areas, and the total power of all frequency bands combined $(0.5-100 \mathrm{~Hz})$. The frequency band of $35-100 \mathrm{~Hz}$ was included to investigate whether the effects to pain were limited to the beta frequencies, or alternatively, whether the adjacent frequency band would show similar effects. No attempt has been made further to resolve the common EEG frequency bands, e.g., a detailed analysis of the delta frequency band.

\section{Statistical analysis}

\section{Study 1}

Intra-individual differences between the perceived pain intensities following hypertonic and isotonic saline injection were examined by means of paired $t$ tests.

\section{Study 2}

Data were summarized using descriptive statistics. A 3-factor repeated measures ANOVA was applied separately for each of the topographic locations. This was based on a study of data from baseline 1, showing that the topographic location had a significant main effect on all EEG parameters. The major experimental factors included: a between-subject factor (experimental sequence based on the randomization of pain and sham pain) and 2 within-subject factors (experimental stages and hemispheres). Greenhouse-Geisser epsilon was used as a correction factor in case the lack of sphericity of the covariance matrix was violated. When the ANOVA $F$ tests proved to be significant, post-hoc tests with Bonferroni's correction were applied to test the significant differences of means among experimental stages. In order to determine whether there is a direct relationship between the power of beta and $35-100 \mathrm{~Hz}$ frequency bands, Pearson's product moment correlation coefficients were computed for each of the topographic locations.

\section{Results}

\section{Characterization of the pain stimulus}

Recorded pain intensities following the injection of hypertonic saline proved to be significantly different from those following the administration of isotonic saline $15 \mathrm{sec}$ after stimulus delivery at the significance level of $P=0.02$, after $30-270 \mathrm{sec}$ at $P<0.01$, and after $235-450 \mathrm{sec}$ at $P<0.05$. Average peak-pain in- tensity following injection of hypertonic saline was 5.2 \pm 2.2 , and $1.7 \pm 1.6$ in the case of isotonic saline.

Based on these data, we employed repeated injections of hypertonic saline as a means to inflict pain for the time needed to obtain sufficient EEG data. Subjects were not informed what time course of pain we aimed to observe. They were advised to report the perceived pain intensity as accurately as possible and that such reporting would be in their own interest. We infused $0.25 \pm 0.07 \mathrm{ml}$ of $5 \%$ saline to achieve the experimental objective.

\section{Intra-individual short-term variability}

The intra-individual short-term variability in topographic EEG measures was assessed in order to determine whether the mean value of selected epochs was suitable for being used as the sample estimate for each of the experimental stages. Eighty-one percent of all observations (740 of 912: 8 topographic locations $\times 6$ EEG parameters $\times 19$ subjects) had CVs of less than 0.20 and $17 \%$ between 0.20 and 0.30 . CVs greater than 0.30 were found in only $2 \%$ of measurements (19 of 912 observations). The majority of the CVs which were greater than 0.30 , in fact, 9 of 19 observations were associated with the $35-100 \mathrm{~Hz}$ frequency band. Notable is the fact that 6 of these 9 measurements were obtained from temporal (T3, T4) recording sites. Of all EEG parameters, the mean frequency showed the lowest magnitude of the CV, with $91 \%$ of the CVs smaller than 0.10 . On the other hand, the power of the delta frequency band demonstrated the highest magnitude of $\mathrm{CVs}$, i.e., $95 \%$ of the CVs in $0.15-0.30$.

\section{Experimental effect on the EEG measures}

Results of the 3-factor repeated measures ANOVA $(2 \times 2 \times 4$ : experimental sequence (based on randomization), hemispheres (right, left), experimental stages (baseline 1: pain, baseline 2: sham pain)) for different topographic locations are listed in Table I. At the probability level of 0.05 , statistically significant interactions were found between hemisphere and experimental stage $(H \times S)$ of the power of the delta frequency band at the frontal recording sites $(\mathrm{F} 3, \mathrm{~F} 4)$ and between hemisphere and experimental sequence $(\mathrm{H} \times \mathrm{G})$ of the power of beta, $35-100 \mathrm{~Hz}$ and $0.5-100 \mathrm{~Hz}$ frequency bands at the parietal recording sites (P3, P4). A significant 3-factor interaction (experimental sequence $x$ hemispheres $x$ experimental stages) was found only for the mean frequency at the temporal recording sites (T3, T4). No significant interactions were found between experimental stage and experimental sequence $(S \times G)$ in any location.

The important result was that for all topographic loci, the experimental stage proved to be a significant main effect for all measurements $(P<0.05)$, except for the power of theta-alpha and delta frequency bands. 
T'ABLE II

DESCRIPTIVE STATISTICS (MEAN, S.D.) OF ALL RECORDED EEG PARAMETERS BY TOPOGRAPHIC LOCI, HEMISPHERES, AND EXPERIMENTAL STAGES $(\mathrm{N}=19$ SUBJECTS $)$

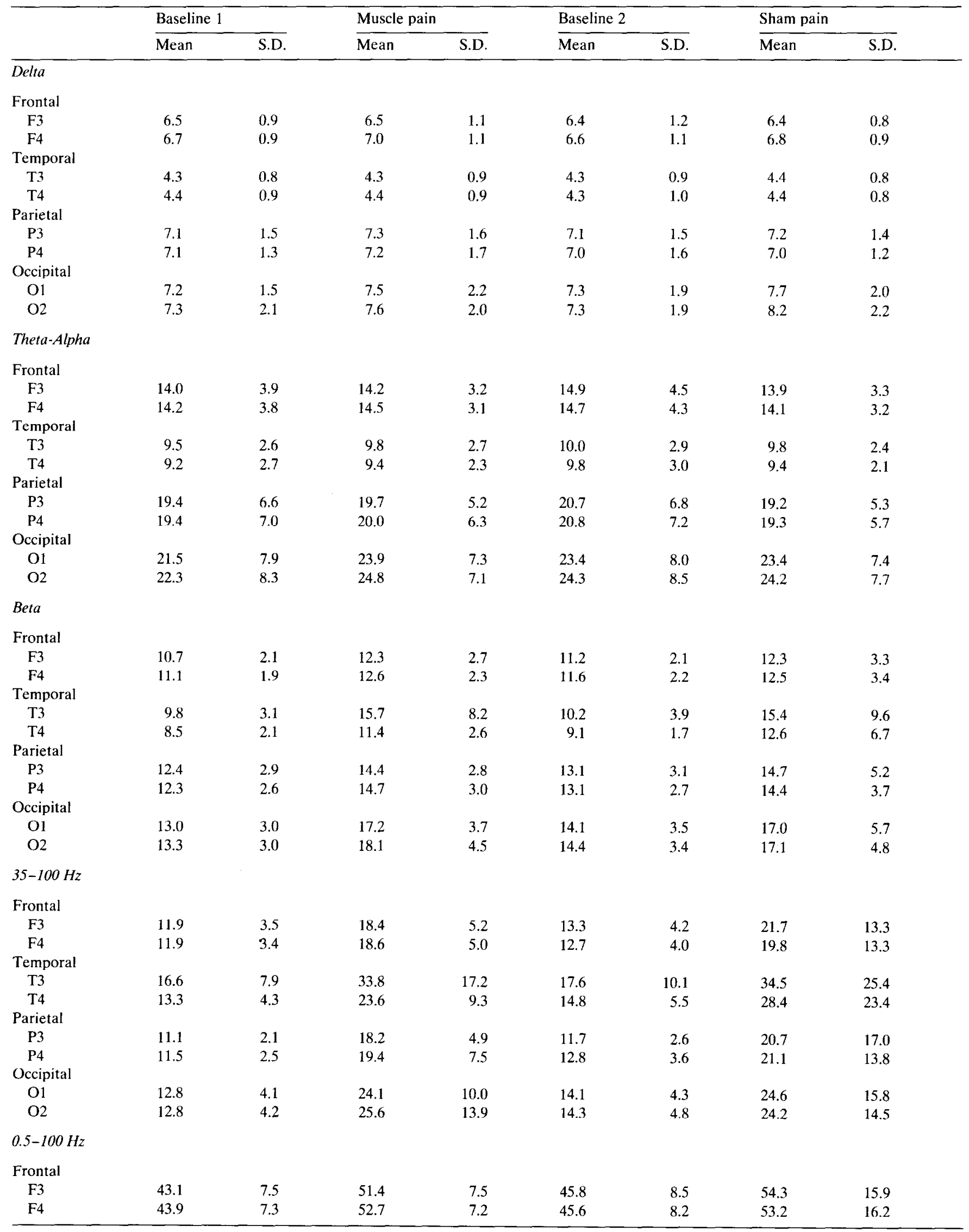


TABLE II (continued)

\begin{tabular}{|c|c|c|c|c|c|c|c|c|}
\hline & \multicolumn{2}{|c|}{ Baseline 1} & \multicolumn{2}{|c|}{ Muscle pain } & \multicolumn{2}{|c|}{ Baseline 2} & \multicolumn{2}{|c|}{ Sham pain } \\
\hline & Mean & S.D. & Mean & S.D. & Mean & S.D. & Mean & S.D. \\
\hline \multicolumn{9}{|c|}{$0.5-100 \mathrm{~Hz}$} \\
\hline \multicolumn{9}{|c|}{ Temporal } \\
\hline $\mathrm{T} 3$ & 40.2 & 10.9 & 63.6 & 25.6 & 42.1 & 14.9 & 64.1 & 35.5 \\
\hline $\mathrm{T} 4$ & 35.4 & 7.5 & 48.8 & 11.4 & 38.0 & 7.0 & 54.8 & 30.1 \\
\hline \multicolumn{9}{|c|}{ Parietal } \\
\hline P3 & 50.0 & 10.6 & 59.6 & 8.8 & 52.6 & 11.1 & 61.8 & 21.4 \\
\hline $\mathrm{P} 4$ & 50.3 & 10.7 & 61.3 & 12.7 & 53.7 & 11.0 & 61.8 & 16.7 \\
\hline \multicolumn{9}{|c|}{ Occipital } \\
\hline $\mathrm{O} 1$ & 54.6 & 13.1 & 72.7 & 14.4 & 58.9 & 13.9 & 72.7 & 22.6 \\
\hline $\mathrm{O} 2$ & 55.7 & 13.8 & 76.1 & 18.3 & 60.3 & 14.3 & 73.7 & 20.0 \\
\hline \multicolumn{9}{|c|}{ Mean Frequency } \\
\hline \multicolumn{9}{|c|}{ Frontal } \\
\hline F3 & 26.7 & 3.8 & 31.6 & 4.6 & 27.8 & 4.5 & 33.1 & 6.6 \\
\hline $\mathrm{F} 4$ & 26.4 & 3.1 & 31.4 & 4.0 & 26.7 & 4.1 & 31.3 & 6.0 \\
\hline \multicolumn{9}{|c|}{ Temporal } \\
\hline $\mathrm{T} 3$ & 33.8 & 5.1 & 41.4 & 5.5 & 33.8 & 5.6 & 40.7 & 6.2 \\
\hline $\mathrm{T} 4$ & 32.6 & 4.4 & 39.1 & 5.4 & 33.4 & 6.1 & 39.1 & 7.3 \\
\hline \multicolumn{9}{|c|}{ Parietal } \\
\hline P3 & 24.0 & 3.2 & 28.8 & 4.4 & 24.1 & 3.5 & 29.3 & 5.9 \\
\hline $\mathrm{P} 4$ & 24.4 & 3.4 & 29.3 & 4.7 & 25.1 & 4.5 & 30.1 & 6.5 \\
\hline \multicolumn{9}{|c|}{ Occipital } \\
\hline $\mathrm{O} 1$ & 24.8 & 3.8 & 30.2 & 6.0 & 25.3 & 4.2 & 29.8 & 4.5 \\
\hline $\mathrm{O} 2$ & 24.6 & 4.5 & 29.8 & 6.1 & 25.2 & 4.7 & 29.4 & 6.1 \\
\hline
\end{tabular}

No significant difference was noted between the left and right hemispheres in any spectral EEG measure except for the power of delta frequency band of the frontal (F3, F4) and the power of beta, $35-100 \mathrm{~Hz}$ and $0.5-100 \mathrm{~Hz}$ frequency bands at the temporal recording sites (T3, T4).

With respect to a between-subject factor, i.e., experimental sequence based on randomization, statistically significant main effects were found only for the power of beta at the parietal (P3, P4) and the beta and $35-100 \mathrm{~Hz}$ frequency bands at the frontal locations (F3, F4). This result suggested that the randomization of the sequential order of the experiment had no major effect on the recorded EEG parameters. As a result, data were pooled.

Mean and standard deviations of all measurements for each experimental stage were computed for all 19 subjects (Table II). Large inter-individual variability was found particularly at the temporal recording sites (T3, T4) for all experimental stages (baselines 1 and 2, experimental pain, and sham pain) and for the following parameters: the power of beta, $35-100 \mathrm{~Hz}$, and total power of the $0.5-100 \mathrm{~Hz}$ frequency bands. The largest subject-to-subject variability was observed in experimental pain and sham pain.

Pooled data from 19 subjects of each experimental stage were subjected to post-hoc analysis. When compared with baseline 1, spectral analyzed EEG during experimental jaw muscle pain and sham pain demon- strated significant increases in power in the $0.5-100 \mathrm{~Hz}$ frequency bands and the mean frequency. This was due to statistically significant higher power in the beta and $35-100 \mathrm{~Hz}$ frequency ranges, most pronounced at the temporal recording sites. There was no statistically significant differences between the measurement parameters for (1) pain vs. sham pain, and (2) baseline 1 vs. baseline $2(P<0.01)$.

In order to demonstrate the variability in the within-subject findings, Fig. 1 summarizes the intra-individual response in the power of all frequency bands at the left temporal recording site (T3) for all subjects and across all experimental stages. Notably, the pattern of power changes in the beta frequency band corresponded with those of the $35-100 \mathrm{~Hz}$ frequency range. This relationship between the power of beta and 35$100 \mathrm{~Hz}$ frequency bands was clearly demonstrated by Pearson's product moment correlation coefficients. At the temporal recording sites, i.e., $\mathrm{T} 3$ and $\mathrm{T} 4$, the correlation coefficients were 0.92 and 0.68 , respectively. For the other recording sites, the correlation coefficients were 0.24 (F3), 0.26 (F4), 0.40 (P3), 0.58 (P4), 0.52 (O1), and $0.77(\mathrm{O} 2)$.

\section{Discussion}

In a single, blind study with subject unaware of the fact that 1 of 2 substances to be administered was 


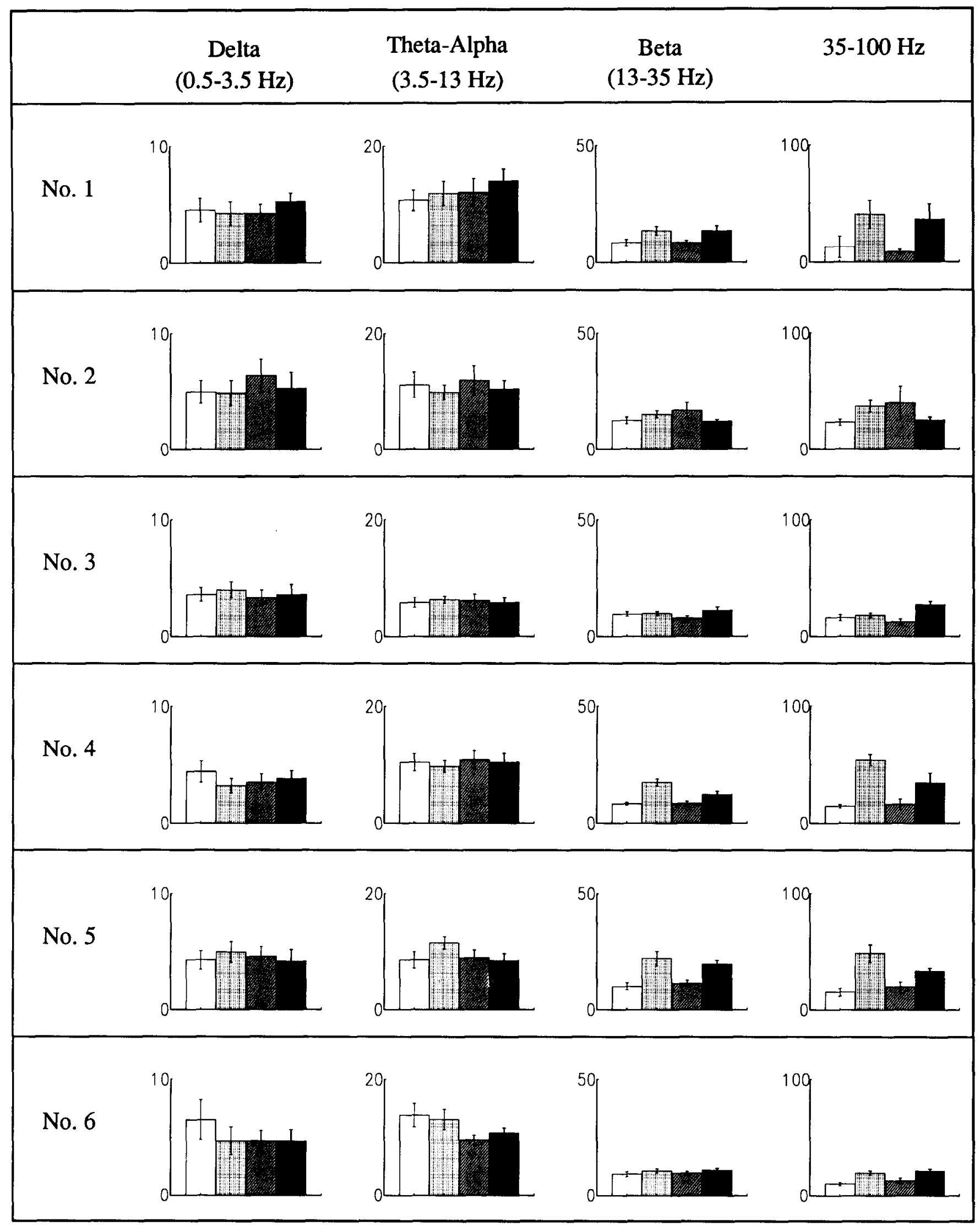

Fig. 1. Intra-individual responses of all 19 subjects in the power ( $\mathrm{nW}$ ) of selected frequency bands for all expcrimcntal stagcs. The error bar represents the standard deviations, computed from 24-30 epochs for each experimental stage. For details, see text. 


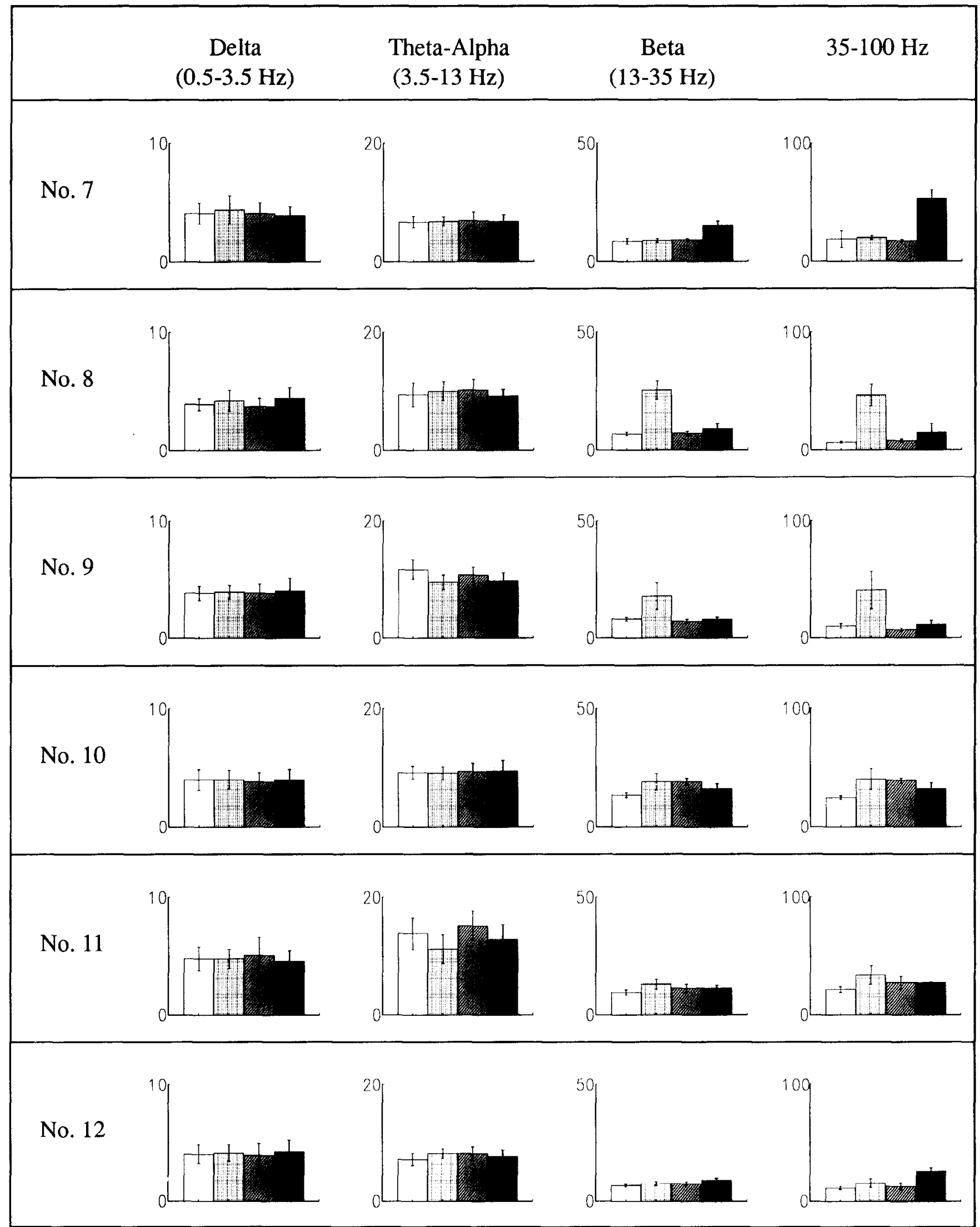




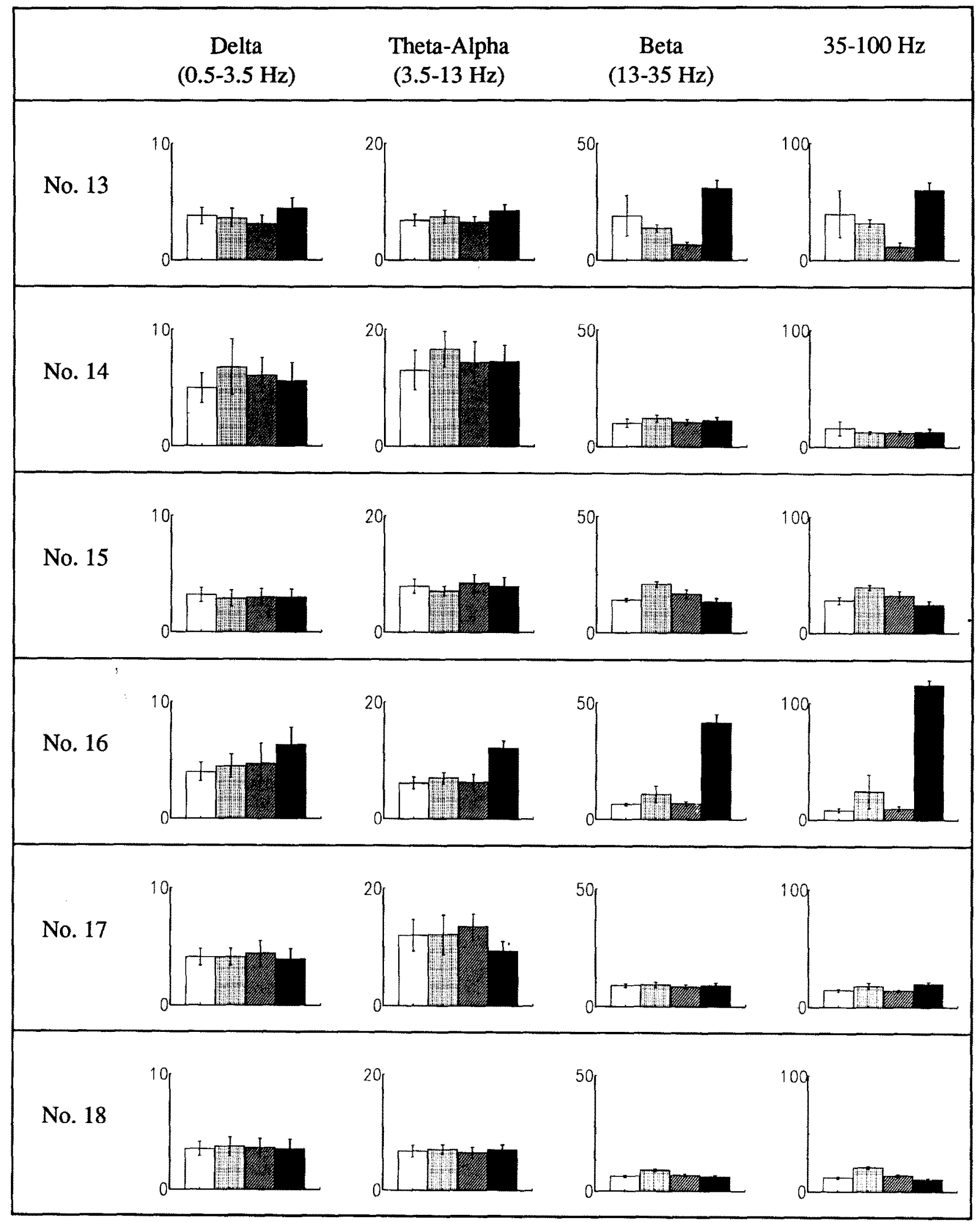

Fig. 1 (continued). 


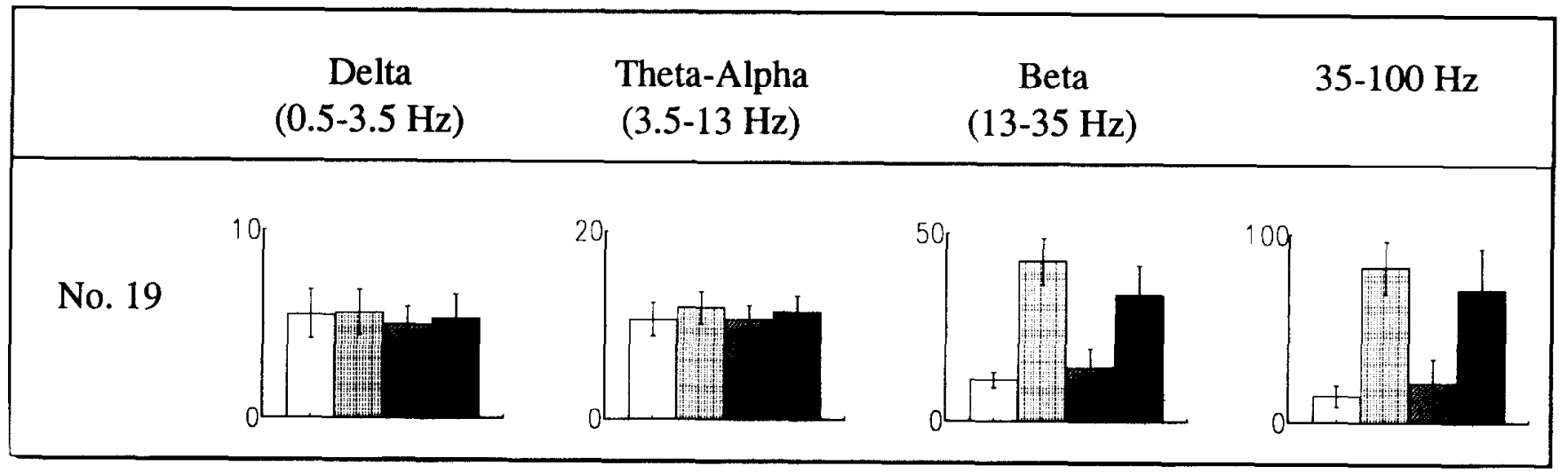

Fig. I (continued).

isotonic saline, we demonstrated that $5 \%$ hypertonic saline was capable of inducing significant pain. This is supported in the literature by earlier reports (Kellgren 1937; Lewis 1938). The minimal pain induced by the injection of isotonic saline was attributed to the needle effect. By means of titration of the stimulus, administering on average $0.25 \pm 0.07 \mathrm{ml}$ in Study 2, we were able to maintain pain for a sufficient amount of time to obtain the needed amount of data for a meaningful comparison of pain and non-pain states. The response not only lasted for a sufficient amount of time but was also quite familiar to our subjects as a "somewhat diffuse" muscle ache (Stohler et al. 1991). This is important in light of the fact that the artificiality of the stimulus is a concern in this kind of experimentation.

In Study 2, we wanted to examine whether the effect of physical pain was any different from pain from memory and whether the two were different from baseline in terms of quantitative EEG measures. The finding of small intra-individual variability at baseline 1 suggested fairly good within-subject consistency. Therefore, we could reliably apply the mean value of selected epochs of each experimental stage for statistical evaluation and hypothesis testing.

Our results indicated that the EEG parameters associated with higher frequencies $(13-100 \mathrm{~Hz})$ were different between non-pain and experimental pain states. Specifically, the statistically significant increase in fast frequency activities was predominantly expressed at the temporal recording sites.

This observation of increase in fast frequencies power during experimental jaw muscle pain is consistent with the prior study using the cold-pressor test (Chen et al. 1989). According to Chen et al. (1989), the significantly heightened cortical beta power density was attributed by the increased vigilance in pain. This assumption is in line with a previous study by Giannitrapani (1971); the increase in beta activity was regarded as a correlate of an internal scanning mechanism of the brain during unstructured stimuli.
Alternatively, the increase in the power of the beta frequency range can be due to contamination from muscle activity during head and body movement (Chen et al. 1983) as well as tonic muscle contraction (O'Donnell et al. 1974). Such activity is difficult to be distinguished or eliminated from EEG records (O'Donnell et al. 1974; Gevins et al. 1977; Gotman et al. 1981; Barlow 1984) and can appear as pseudo-beta activity (Barlow 1984). Based on this information, the origin, brain or muscle, of increased beta spectral power during painful condition should be called into question.

In order to provide further insight into this question, we also analyzed the spectral power of higher-thancommon EEG frequency bands, i.e., $35-100 \mathrm{~Hz}$. As our results indicated, the spectral power in the 35-100 $\mathrm{Hz}$ domain demonstrated a significant increase during experimental pain and correlated well with the changes in the beta frequency band. This phenomenon was particularly expressed at the temporal recording sites with electrodes located directly over facial and jaw muscles. In addition, the observed significant shift of mean frequency toward higher frequencies also suggested the possibility of contamination from cranio-facial muscles. There is some evidence supporting our suggestion. Studies using video recordings demonstrated changes of facial expression in response to clinical and experimental pain (LeResche et al. 1984; Craig and Patrick 1985; LeResche and Dworkin 1988) and the scalp electromyographic activity is altered by experimental pain (Schimek et al. 1984). Moreover, the facial electromyographic response is correlated with the emotional state (cf., Cacioppo et al. 1986; Dimberg 1990).

In light of these findings, our observations are strongly suggestive of the contamination of EEG data from activities of the psychomotor loop most notably affecting the beta frequency band. In addition, the finding of significant inter-individual differences between hemispheres on the temporal locations can also be interpreted as evidence of a specific within-subject 
cranio-facial muscle response rather than due to structured, functional organization of the brain. The unexplained variance in the heightened beta cortical power density can be attributed to the vigilance scanning of pain processes as suggested by Chen et al. (1989).

In agreement with Chen et al. (1989), there was no significant increase in the theta-alpha power during experimental pain state. In our study, however, no significant change was found in the power of the delta band $(0.5-3.5 \mathrm{~Hz})$. In this respect, we are unable to compare our findings with the work of Chen et al. (1989), reporting a dichotomy of the response of paintolerant and pain-sensitive subjects as far as the delta frequencies are concerned.

With respect to sham pain, statistically significant increases in the power of fast frequencies were observed as well. However, the EEG measures of sham pain did not differ statistically to a significant degree from those of experimental pain. This effect can be explained on the basis of a psychophysiological response. According to Lang (1979), image processing can produce physiological responses in the same fashion as the actual perception. During imagination of a fearful scene, a change in heart rate, respiratory rate, muscle activity and eye movement is observed (Jones and Johnson 1980; Lang et al. 1980; Schwartz et al. 1980). The vividness of the image (clear, fair, vague) and the type of image task (fear, neutral, anxiety, fantasy, etc.) (Lang 1979) are likely to contribute to the observed inter-individual variability. Based on the fact that we could not observe a statistically significant difference in topographic EEG measures in pain versus imagined pain, we concluded that the observed effects in the beta frequency band are non-specific for pain.

\section{Acknowledgment}

We wish to thank Charles J. Kowalski, Ph.D., Statistical Research Unit, School of Dentistry, and Kathleen Welch, Statistical Research Laboratory, The University of Michigan, for their helpful suggestions. This study was supported by USPH NIH-NIDR RO1-DE8606-04.

\section{References}

Barlow, J.S., EMG artifact minimization during clinical EEG recordings by special analog filtering, Electroenceph. Clin. Neurophysiol., 58 (1984) 161-174.

Bromm, B., Evoked cerebral potential and pain, Adv. Pain Res. Ther., (1985) 305-329.

Cacioppo, J.T., Petty, R.E., Losch, M.E. and Kim, H.S., Electromyographic activity over facial muscle regions can differentiate the valence and intensity of affective reactions, J. Pers. Soc. Psychol., S0 (1986) 260-268.
Carmon, A., Mor, J. and Goldberg, J., Evoked cerebral responses to noxious thermal stimuli in humans, Exp. Brain Res., 25 (1976) 103-107.

Carmon, A., Dotan, Y. and Sarne, Y., Correlation of subjective pain experience with cerebral evoked responses to noxious thermal stimulations, Exp. Brain Res., 33 (1978) 445-453.

Chapman, C.R., Chen, A.C.N. and Harkins, S.W., Brain evoked potentials as correlates of laboratory pain: a review and perspective, Adv. Pain Res. Ther., 3 (1979) 791-803.

Chapman, C.R., Chen, A.C.N., Colpitts, Y.M. and Martin, R.W., Sensory decision theory described evoked potential in pain discrimination, Psychophysiology, 18 (1981) 114-120.

Chatrian, G.E., Canfield, R.C., Knauss, T.A. and Lettich, E., Cerebral responses to electrical tooth pulp stimulation in man, Neurology (Minn.), 25 (1975) 745-757.

Chen, A.C.N., Chapman, C.R. and Harkins, S.W., Brain evoked potentials are functional correlates of induced pain in man, Pain, 6 (1979) 365-374.

Chen, A.C.N., Dworkin, S.F. and Bloomquist, D.S., Cortical power spectrum analysis of hypnotic pain control in surgery, Intern. J. Neurosci., 13 (1981) 127-136.

Chen, A.C.N., Drangsholt, M.T., Dworkin, S.F. and Clark, D.W., Microcomputer analysis of cortical power spectrum calibration and correlates of behavioral artifacts, Biol. Psychol., 16 (1983a) $181-196$.

Chen, A.C.N., Dworkin, S.F. and Drangsholt, M.T., Cortical power spectral analysis of acute pathophysiological pain, Intern. J. Neurosci., 18 (1983b) 269-278.

Chen, A.C.N., Dworkin, S.F., LeResche, L. and Truelove, E., Alpha activity and acute pathophysiological pain: cortical power spectrum analysis, Pain, Suppl. 2 (1984) abst. 244.

Chen, A.C.N., Dworkin, S.F, Haug, J. and Sommers, E., Human pain responsiveness: benzodiazepine vs. endorphine action on cortical power spectrum analyses, Pain, Suppl. 2 (1987) abst. 63.

Chen, A.C.N., Dworkin, S.F., Haug, J. and Gerig, J., Topographic brain measures of human pain and pain responsivity, Pain, 37 (1989) 129-141.

Chudler, E.H. and Dong, W.K., The assessment of pain by cerebral evoked potentials, Pain, 16 (1983) 221-244.

Craig, K.D. and Patrick, C.J., Facial expression during induced pain, J. Pers. Soc. Psychol., 48 (1985) 1080-1091.

Cruccu, G., Fornarelli, M., Inghilleri, M. and Manfredi, M. The limits of tooth pulp evoked potentials for pain quantitication, Physiol. Behav ${ }_{x,} 31$ (1983) 339-342.

Desmedt, J.E. and Debecker, J., Slow potential shifts and decision $\mathrm{P} 350$ interactions in task with random sequences of near-threshold clicks and finger stimuli delivered at regular intervals, Electroen. ccph. Clin. Neurophysiol., 47 (1979) 671-679.

Dimberg, U., Facial electromyography and emotional reactions, Psychophysiology, 27 (1990) 481-494.

Drake, M.E., J.R.C., Craig Du Bois, Huber, S.J., Pakalnis, A. and Denio, L.S., EEG spectral analysis and time domain descriptions in headache. Headache, 28 (1988) 201-203.

Dworkin, S.F., Chen, A.C.N., LeResche, L. and Truelove, E., Perception of acute pain and changing levels of chronic orofacial pain: pain assessment and preliminary EEG findings, Pain, Suppl. 2 (1984) abst. 604.

Gevins, A.S., Yeager, C.L., Zeitlin, G.M., Ancoli, S. and Dedon, M.F., On-line computer rejection of EEG artifact, Electroenceph. Clin. Neurophysiol., 42 (1977) 267-274.

Giannitrapani, D., Scanning mechanisms and the EEG, Electroenceph. Clin. Neurophysiol., 30 (1971) 139-146.

Gotman, J., Ives, J.R. and Gloor, P., Frequency content of EEG and EMG at seizure onset: possibility of removal of EMG artifact by digital filtering, Electroenceph. Clin. Neurophysiol., 38 (1981) 623-639. 
Harkins, S.W. and Chapman, C.R., Cerebral evoked potentials to noxious dental stimulation: relationship to subjective pain report, Psychophysiology, 15 (1978) 248-252.

Jones, G.E. and Johnson, H.J., Heart rate and somatic concomitants of mental imagery, Psychophysiology, 17 (1980) 339-347.

Kellgren, J.H., Observations on referred pain arising from muscle, Clin. Sci., 3 (1937) 175-190.

Lang, P.J., A bio-informational theory of emotional imagery, Psychophysiology, 16 (1979) 495-512.

Lang, P.J., Kozak, M.J., Miller, G.A., Levin, D.N. and McLean, A., Jr., Emotional imagery: conceptual structure and pattern of somato-visceral response, Psychophysiology, 17 (1980) 179-192.

Leandri, M., Campbell, J.A. and Lahuerta, J., Effect of attention on tooth-pulp evoked potentials, Adv. Pain Res. Ther., 9 (1985) $331-336$.

LeResche, L. and Dworkin, S.F., Facial expressions of pain and emotions in chronic TMD patients, Pain, 35 (1988) 71-78.

LeResche, L., Dworkin, S.F. and Chen, A.C.N., Relationship between facial expression and self report of acute dental pain, Pain Suppl. 2 (1984) abst. 196.

Lewis, T., Suggestions relating to the study of somatic pain, Br. Med. J., 1 (1938) $321-325$.

Manna, V., Negro, F.E., Rinaldi, R. and Varrassi, G., Effects of acupuncture on subjective pain, psychological tests and spectral analysis EEG in patients affected hy tension headache, Pain, Suppl. 2 (1984) abst. 226.

O'Donnell, R.D., Berkhout, I. and Adey, W.R., Contamination of scalp EEG spectrum during contraction of cranio-facial muscles, Electroenceph. Clin. Neurophysiol., 7 (1974) 145-151.

Schimek, F., Gundling-Hoffman, A., Ebeling, J. and Schorer, R., The effect of pain on scalp EMG, Pain, Suppl. 2 (1984) abst. 245.

Schwartz, G.E., Brown. S.-L. and Ahern, G.L., Facial muscle patterning and subjective experience during affective imagery: sex differences, Psychophysiology, 17 (1980) 75-82.

Stohler, C.S., Zhang, X. and Ashton-Miller, J.A., An experimental model of jaw muscle pain in man. In: Z. Davidovitch (Ed.). The Biological Mechanisms of Tooth Movement and Craniofacial Adaptation, EBSCO Media. Birmingham, 1991, pp. 261-267.

Velasco, M., Velasco, F., Machado, J. and Olivera, A., Effects of novelty, habituation, attention and distraction on the amplitude of the various components of the somatic evoked responses, Intern. J. Neurosci., 5 (1973) 101-111.

Zhang, X., Ashton-Miller, J.A. and Stohler, C.S., A closed-loop system for maintaining experimental muscle pain in man, IEEE Trans. Biomed. Eng., submitted. 